\title{
Oseltamivir-induced bradycardia in an adult patient
}

\begin{abstract}
The use of Oseltamivir, the first neuraminidase inhibitor available orally, has increased tremendously since the first pandemic of H1N1 influenza virus in 2009, as a mean of prevention as well as treatment of influenza. Its use has been associated with common side effects such as nausea and vomiting. However, few data is published concerning cardiovascular side effects that could lead to some serious complications. This case report presents a young healthy woman who developed sinus bradycardia while being on Oseltamivir therapy.
\end{abstract}

Volume 7 Issue 4 - 2019

\author{
Krystel Chahine, ${ }^{2}$ Yahya Soufi, ${ }^{2}$ Chantal \\ Chahine, ${ }^{2}$ Tony El Murr' \\ 'Specialist in Internal medicine and Clinical Immunology, Head of \\ medicine Department, Middle East Institute of Health University \\ Hospital, Lebanon \\ ${ }^{2}$ Faculty of Medical Science, Lebanese university, Lebanon
}

\begin{abstract}
Correspondence: Tony El Murr, Specialist in Internal medicine and Clinical Immunology, Head of medicine Department, Middle East Institute of Health University Hospital Bsalim, Lebanon, Tel +(961)3347473, Email drtonimurr@hotmail.com
\end{abstract}

Received: June 14, 2019 | Published: July 04, 2019

\section{Introduction}

While most cases of H1N1 are self-limiting, some cases might be life threatening, especially in extremes of ages, pregnant, immunocompromised and patients with co-morbidities. The use of Oseltamivir, a neuraminidase inhibitor, for the prevention as well as treatment of influenza has increased tremendously since the first pandemic of H1N1 influenza virus in 2009. ${ }^{1}$ However, its use has been associated with commonly reported side effects such as headache, nausea, vomiting, ${ }^{2}$ as well as few cases of cardiac rhythm abnormalities. ${ }^{3}$ Keeping this background in mind, we report a case of sinus bradycardia in a patient while being on oseltamivir therapy for H1N1 influenza.

\section{Case report}

A 28-year-old woman, non smoker, non alcohol consumer, known to have epilepsy on depakine and levipram, was hospitalized with a presumptive diagnosis of pneumonia and was started on levofloxacin. However, patient remained febrile and developed desaturation 48 hours post admission, for which she required intubation and ICU transfer. CT chest showed multilobar pneumonia. H1N1 influenza was suspected and was subsequently validated by PCR. Oseltamivir $75 \mathrm{mg}$ twice daily was initiated in association with clarithromycin, vancomycin, meropenem and bronchodilator inhalations. The admission heart rate was $124 \mathrm{bpm}$, decreasing to $52 \mathrm{bpm}$ the morning following oseltamivir initiation; Patient experienced repeated episodes of bradycardia reaching $42 \mathrm{bpm}$ which required external pacemaker placement at the $6^{\text {th }}$ day in the ICU. Fever had by then resolved and patient was extubated; previous antibiotics and oseltamivir were stopped and she was started again on levofloxacin with no recurrence of bradycardia. Her subsequent stay in hospital was uneventful. Heart rate improved and temporary pacemaker was removed after 5 days of observation. Note that of the patients' medications other than oseltamivir; none is known to be associated with bradycardia. Thyroid function, cardiac enzymes, and echocardiography done were normal.

\section{Discussion}

Oseltamivir carboxylate is an inhibitor of influenza virus neuraminidase, acting through the inhibition of viral particle release. This antiviral is used for prophylaxis and treatment of Influenza A and Influenza B in adults, adolescents, and pediatric populations. ${ }^{4}$
However, its use has been associated with psychiatric reactions, renal, metabolic (hyperglycemia or diabetes), and cardiac reactions (QT prolongation) related to Oseltamivir's interference with human endogenous neuraminidase. ${ }^{5}$ In fact, after being converted into its active metabolite in the GI tract and liver, Oseltamivir reaches a steady-state plasma concentration within 2-3 days of twice-daily administration. This active metabolite has a plasma half-life $\left(\mathrm{t}_{1 / 2}\right)$ of 6-10 hours. The bradycardia as an Adverse Drug Reaction (ADR) was observed within 2-3 days of the beginning of the therapy which coincided with the steady state concentration of Oseltamivir in the body. Abatement of ADR was seen after 3-6 days coinciding with the time to elimination of the drug from the body. ${ }^{6}$ A systemic review in Vigi Access database showed 13 cases of bradycardia out of total 12141 ADRs of Oseltamivir $(0.10 \%)$ and in eHealthME database, 122 bradycardia cases out of 14220 ADRs of Oseltamivir $(0.86 \%) .{ }^{1}$ Although Oseltamivir-induced bradycardia is considered rare, it is crucial to recognize it in order to avoid further harm, especially if the patient has a history of cardiac disease or is on bradycardic cardiovascular medications. Having this in mind helps also physicians minimize time consuming bradycardia investigations, as it seems that this drug's adverse effect is reversible with no effect on patients' hemodynamics.

\section{Conclusion}

The case presented demonstrates a potential link between Oseltamivir and bradycardia in the adult population. Physicians should have in mind the trade-off between benefits and harms when prescribing oseltamivir as prophylaxis or treatment for influenza, and a high index of suspicion for this possible side effect whenever they face a non-explained bradycardia in patients treated by Oseltamivir.

\section{Acknowledgments}

None.

\section{Conflicts of interest}

Authors declare that there is no conflict of interest.

\section{References}

1. Shah KB, Shah BS, Malhotra SD, et al. Oseltamivir induced sinus bradycardia: an area of potential concern. International Journal of Basic and Clinical Pharmacology. 2019;8(1):162-165. 
2. Jefferson T, Jones MA, Doshi P, et al. Neuraminidase inhibitors for preventing and treating influenza in healthy adults and children. Cochrane Database Syst Rev. 2014;4(4):CD008965.

3. Rossi S. Australian Medicines Handbook. 7th ed. Sydney; 2006. 143-145 p.

4. Arabi H, Zaid AA, Alreefi M, et al. Suspected Oseltamivir-induced bradycardia in a pediatric patient: A case report from King Abdullah Specialist Children's Hospital, Riyadh, Saudi Arabia. Clin Pract. 2018;8(4):1094.
5. Hama R. The mechanisms of delayed onset type adverse reactions to oseltamivir. Infect Dis (Lond). 2016;48(9):651-660.

6. Davies BE. Pharmacokinetics of oseltamivir: an oral antiviral for the treatment and prophylaxis of influenza in diverse populations. $J$ Antimicrob Chemother. 2010;65(suppl_2):ii5-ii10. 